\title{
COVID-19 pneumonia: infection control protocol inside computed tomography suites
}

\author{
Kento Nakajima ${ }^{1} \cdot$ Hideaki Kato $^{2} \cdot$ Tsuneo Yamashiro $^{3,6} \cdot$ Toshiharu Izumi $^{4} \cdot$ Ichiro Takeuchi $^{1} \cdot$ Hideaki Nakajima $^{5}$. \\ Daisuke Utsunomiya ${ }^{3,4}$
}

Received: 28 February 2020 / Accepted: 5 March 2020 / Published online: 17 March 2020

(c) Japan Radiological Society 2020

\begin{abstract}
A novel coronavirus (severe acute respiratory syndrome coronavirus 2) causes a cluster of pneumonia cases in Wuhan, China. It spread rapidly and globally. CT imaging is helpful for the evaluation of the novel coronavirus disease 2019 (COVID-19) pneumonia. Infection control inside the CT suites is also important to prevent hospital-related transmission of COVID-19. We present our experience with infection control protocol for COVID-19 inside the CT suites.
\end{abstract}

Keywords COVID-19 · Pneumonia · Computed tomography · Infection

A novel coronavirus, severe acute respiratory syndrome coronavirus 2 (SARS-CoV-2), was identified as the etiology for a cluster of pneumonia cases in Wuhan, a city in the Hubei Province of China [1]. It spread rapidly throughout China, resulting in a nation-wide epidemic; moreover, there were reports of sporadic cases globally. Previously published reports describe the imaging features of patients with the novel coronavirus disease 2019 (COVID-19) pneumonia $[2,3]$. These reports have highlighted the clinical importance of chest computed tomography (CT) for the detection and management of patients infected with SARS-CoV-2. On the other hand, the infection control in the CT suite is also critically important because high rate of presumed

Daisuke Utsunomiya

d.utsunomiya@gmail.com

1 Advanced Critical Care Medicine, Yokohama City University Hospital, Yokohama, Japan

2 Infection Prevention and Control Department, Yokohama City University Hospital, Yokohama, Japan

3 Diagnostic Radiology, Yokohama City University Hospital, 3-9, Fukuura, Kanazawa-ku, Yokohama, Kanagawa 236-0004, Japan

4 Central Radiology, Yokohama City University Hospital, Yokohama, Japan

5 Hematology and Clinical Immunology, Yokohama City University Hospital, Yokohama, Japan

6 Department of Radiology, Graduate School of Medical Science, University of the Ryukyus, Nishihara, Japan hospital-related transmission of COVID-19 (41.3\%) was reported $[1,4]$. Radiologists should not only be aware of the CT features of patients with COVID-19 pneumonia but they must also be well informed of the infection control protocols to be followed inside the CT suite to prevent the spread of infection. Herein, we present our experience with infection control protocol for COVID-19 inside the CT suite.

\section{Personal protective equipment (PPE)}

Two radiologic technologists (RTs) are desirable for the CT scanning of patients for the assessment of COVID-19 pneumonia. One RT uses PPE to set up the patient on the CT imaging table, while the other RT operates the CT console. The PPE includes eye protection (goggles), a filtering facepiece respirator (N95), a surgical cap, gloves, a fluidresistant gown, and shoe covers (Fig. 1).

\section{Protocol for chest CT imaging at CT suite}

In principle, CT examinations for patients suspected of or confirmed with SARS-CoV-2 infection are performed last in the daily CT schedule. The schematic of our CT suite is shown in Fig. 2. The CT examination is performed as follows (Fig. 3): (1) RT-1 with PPE is on stand-by; (2) the patient and the attending medical doctor (MD) arrive inside the CT scanner room; (3) RT-1 and MD set up the patient 

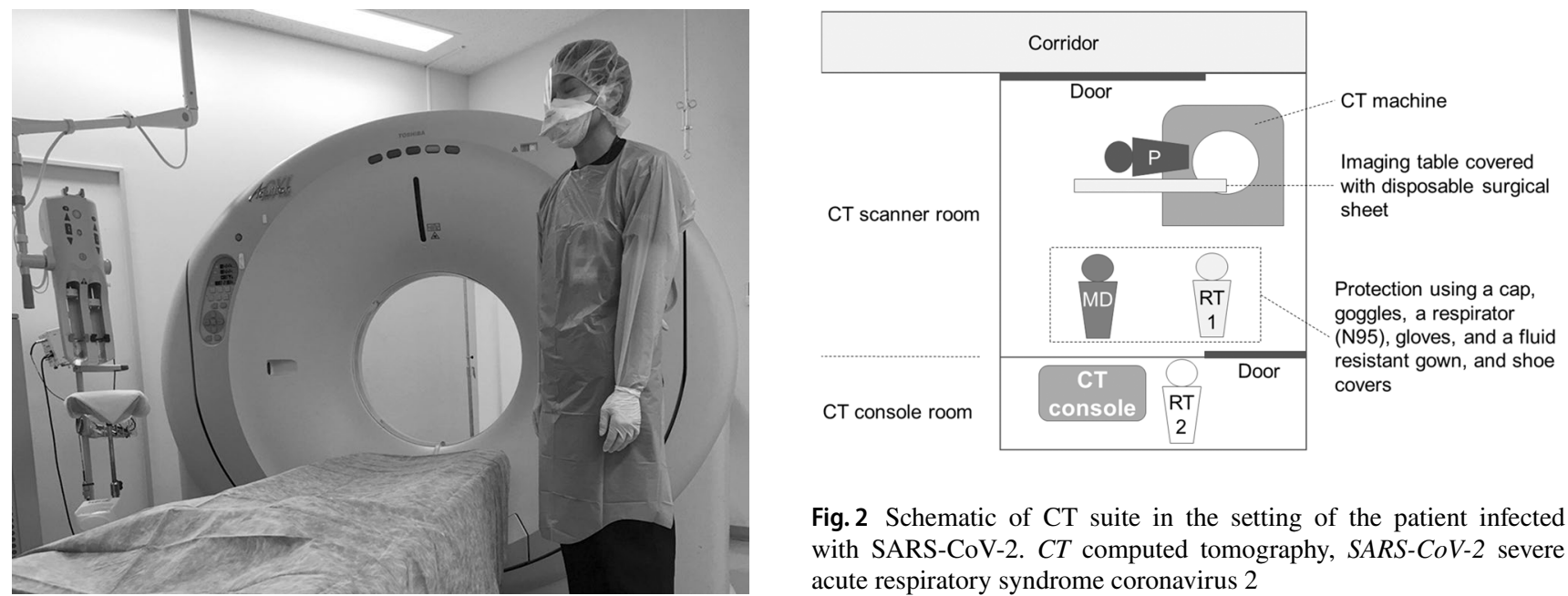

Fig. 2 Schematic of CT suite in the setting of the patient infected with SARS-CoV-2. CT computed tomography, SARS-CoV-2 severe acute respiratory syndrome coronavirus 2

Fig. 1 Personal protective equipment (a radiologic technologist)

Fig. 3 Process and staff distribution in the CT scanner and console rooms during CT examination of the patient infected with SARS-CoV-2. $R T$ radiologic technologist, $P$ patient, $M D$ medical doctor, $C T$ computed tomography, SARS$\mathrm{CoV}-2$ severe acute respiratory syndrome coronavirus 2

\begin{tabular}{|c|c|c|c|}
\hline & CT scanner room & \multicolumn{2}{|c|}{ CT console room } \\
\hline \multicolumn{4}{|l|}{ Stand-by } \\
\hline \multicolumn{4}{|l|}{$\begin{array}{l}\text { Patient Arrival \& } \\
\text { Setting }\end{array}$} \\
\hline \multicolumn{4}{|l|}{ After Setting } \\
\hline Scanning & & $\begin{array}{l}\text { MD put on } \\
\text { PPE. }\end{array}$ & $\begin{array}{l}\text { RT2 performs } \\
\text { CT scan. }\end{array}$ \\
\hline $\begin{array}{l}\text { After Scanning \& } \\
\text { Leaving }\end{array}$ & & & \\
\hline
\end{tabular}

on the CT imaging table; (4) RT-1 and MD remove and discard their contaminated PPE (RT-1 and MD) inside the CT suite and perform hand hygiene; (5) RT-1 and MD leave the CT scanner room; (6) RT-2 performs non-contrast chest CT scanning, while RT-1 and MD put on new PPE outside the CT scanner room; (7) after the CT scanning, RT-1 and MD transfer the patient from the CT imaging table; (8) the patient and MD leave the CT scanner room; (9) the CT scanner and console rooms are sanitized. After leaving the CT suite, the high-frequency contact surfaces (i.e., operating consoles, switches, handles, and door knobs) are wiped with a cloth soaked with alcohol-based disinfectants. The CT suite is tightly closed for $1 \mathrm{~h}$ to thoroughly ventilate and exchange the room air.
We use a 64-detector CT (SOMATOM definition AS+, Siemens) with the following scanning parameters: collimation, $128 \times 0.6 \mathrm{~mm}$; gantry rotation speed, $0.5 \mathrm{~s}$; X-ray tube voltage, $120 \mathrm{kV}$ with automatic tube current modulation. The CT images are reconstructed with a slice thickness of $2 \mathrm{~mm}$ and increment of $2 \mathrm{~mm}$. Coronal and sagittal multiplanar reconstruction images are also acquired.

\section{References}

1. Wang D, Hu B, Hu C, Zhu F, Liu X, Zhang J, et al. Clinical characteristics of 138 hospitalized patients with 2019 novel coronavirus-infected pneumonia in Wuhan, China. JAMA. 2020. https:// doi.org/10.1001/jama.2020.1585. 
2. Pan Y, Guan H, Zhou S, Wang Y, Li Q, Zhu T, et al. Initial CT findings and temporal changes in patients with the novel coronavirus pneumonia (2019-nCoV): a study of 63 patients in Wuhan, China. Eur Radiol. 2020. https://doi.org/10.1007/s00330-02006731-x.

3. Pan F, Ye T, Sun P, Gui S, Liang B, Li L, et al. Time course of lung changes on chest CT during recovery from 2019 novel coronavirus (COVID-19) pneumonia. Radiology. 2020. https:// doi.org/10.1148/radiol.2020200370.
4. Japanese College of Radiology. Introduction of infection protection in CT scanning in case with novel coronavirus pneumonia. https://jcr.or.jp/2020/02/22/01/. Accessed 27 Feb 2020.

Publisher's Note Springer Nature remains neutral with regard to jurisdictional claims in published maps and institutional affiliations. 